\title{
ONE-DIMENSIONAL EXCITON FUSION KINETICS IN DILUTE POLYMER BLENDS
}

\author{
R. KOPELMAN, C.S. LI and Z.-Y. SHI \\ Department of Chemistry, University of Michigan, Ann Arbor, MI 48109, USA
}

\begin{abstract}
Exciton-exciton and exciton-excimer triplet fusion kinetics is monitored in medium molecular weight P1VN/PMMA films with concentrations from 0.005 to $100 \%$ (weight), at temperatures of 77 to $300 \mathrm{~K}$, via time-resolved fluorescence and phosphorescence (10 ns to $10 \mathrm{~s}$ ). The triplet-triplet annihilation is bimolecular at short times but pseudo-monomolecular at long times. Furthermore, the heterogeneity exponent $(h)$ is 0.5 for isolated P1VN chains, zero (classical) for pure P1VN and "fractal-like" $(0<h<0.5)$ throughout certain concentration regimes. However, $h$ is not monotomic with blend concentration but rather oscillates between zero and 0.5 . Correlation is made with morphology changes (phase separation, filamentation). The long-lived decays do fit stretched exponentials, with a parameter $\beta=1-h$. Distinction is also made between diffusionlimited and reaction-limited kinetic regimes. Furthermore, the blend topology is also studied with the aid of the time-modulation technique, in which the time decays are obtained for different excitation durations, i.e., single pulse vs. cw or multiple-pulse laser excitation, but with equal global exciton densities at the start $(t=0)$ of the decays. As expected, the triplet exciton kinetics is dominated by short-range hops (about $5 \AA$ ) and thus monitors the primary topology of the chains. At concentrations below $0.01 \%$, the excitons are constrained to a truly one-dimensional topology. This is probably the most ideal case of a molecular wire conducting elementary excitations.
\end{abstract}

\section{Introduction}

New analytical theory, computer simulations and experiments on model systems have led to new concepts of reaction kinetics in low and restricted dimensions [1]. This, in turn, has led to new experimental characterizations of composite materials, such as polymer blends $[2,3]$ and molecularly doped polymers [4,5]. The morphology of these disordered materials is studied with the help of excitation kinetics. Using triplet excitation transport and fusion, the relevant matrix elements are extremely short-ranged. This enables one to obtain morphological information on the scale of the monomer units.

\section{Experimental}

Both P1VN (MW = 100000) and PMMA (MW = 154000 ) used in this study are purchased from Polyscience Inc. They are all purified and prepared by a detailed procedure [2]. The thickness of the finished film is about $20 \mu \mathrm{m}$.

When experiments are performed at liquid nitrogen temperature $(77 \mathrm{~K})$, the samples are immersed in a liquid nitrogen bath inside a quartz cryostat. Alternatively, the samples are placed in the same cryostat, and room temperature nitrogen gas is kept flowing. This eliminates oxygen quenching of the triplets. Excitation light for this study is a Lambda Physics Excimer laser, with $\mathrm{XeCl}$; it gives about a 10 ns pulse at the wave- length of $308 \mathrm{~nm}$ and the linewidth is severeal angstroms. Usually, the output power of the laser is much stronger than required; therefore, neutral density filters are used. For consistency, each set of experiments is performed at the same strength of excitation light.

For the kinetic studies the samples inside the cryostat are usually placed at $45^{\circ}$ to the incident excitation and the emission signals are picked up by 2 UV grade lenses, which focus the light on the entrance slit of a SPEX 0.5 M double spectrometer. In order to pick up more signal and increase the signal-to-noise ratio, both the entrance and exit slits are open wide (about 1000 $\mu \mathrm{m}$ or more). For phosphorescence decay signals the monochromator is set at $5250 \AA$, while for the delayed fluorescence the signals are collected at around $3400 \AA$. These settings are based on the steady-state emission spectra of P1VN/PMMA. At the exit slit a regular EMI photomultiplier tube (PMT) is attached. The samples with concentrations lower than $2 \%$, due to the fact that the decay signals are weak and the films are relatively clear, are placed at $90^{\circ}$ to the excitation light. The emission signals are collected at the back of the sample (similarly to the way in which the absorption spectrum is taken). Both delayed fluorescence and phosphorescence decay emissions are first passed through a $\mathrm{CuSO}_{4}$ solution $\left(100 \mathrm{~g} \mathrm{CuSO}_{4} \cdot 5 \mathrm{H}_{2} \mathrm{O}\right)$ to filter out the residual laser light. Then a $340 \mathrm{~nm}$ band pass filter is used before the PMT to collect the delayed fluorescence. For phosphorescence decay at $520 \mathrm{~nm}$ band pass filter is used. The current output from the PMT is fed into a Signal Averager (Princeton Applied Research Model 
4202). The termination resistor is selected to be small enough to eliminate the intrinsic effect of the apparatus on the fast decay of the samples. A Wavetek Model 187 function generator provides the external trigger. For the early time decay data collections, the rising edge of an $\sim 40 \mu \mathrm{s}$ wide pulse is used to trigger the excimer laser, while the falling edge of the pulse is used to trigger the signal averager. (The pulse-width is selected as a parameter to exclude the residual laser light which is reflected into the spectrometer for the higher concentration samples.) With the above adjustments and the resolution of the signal averager, no signal is detected with a blank stainless steel plate. Therefore, all the decay data reported here for the early time kinetics have a $40 \mu \mathrm{s}$ offset. For the longer time kinetics studies the data are collected about $10 \mathrm{~ms}$ after the laser fires.

\section{Results and discussion}

The longer time data are fit by the expression [6]

$I_{\mathrm{DF}}=k I_{\mathrm{p}}, \quad k=k_{0} t^{-h}$,

relating the delayed fluorescence to the phosphorescence via a time-dependent rate coefficient [1,2]. The "heterogeneity exponent" $h$ describes the effective topology of the triplet excitation transport, resulting in the (diffusion-controlled) exciton fusion, triplet + triplet $\rightarrow$ singlet, responsible for the delayed fluorescence. Figure 1 shows the results for a very dilute sample $(0.01$ wt\% P1VN/PMMA). The slope gives $h=0.53 \pm 0.03$, in excellent agreement with the theoretical expectation for one-dimensional topology $\left(h=\frac{1}{2}\right)$. We note that the triplet excitation transport (in contrast to singlet transport), is extremely short-ranged ( $3-4 \AA$ ), limiting excitation hopping to adjacent monomers (unless non-adjacent ones come extremely close). Thus, for triplet excita-

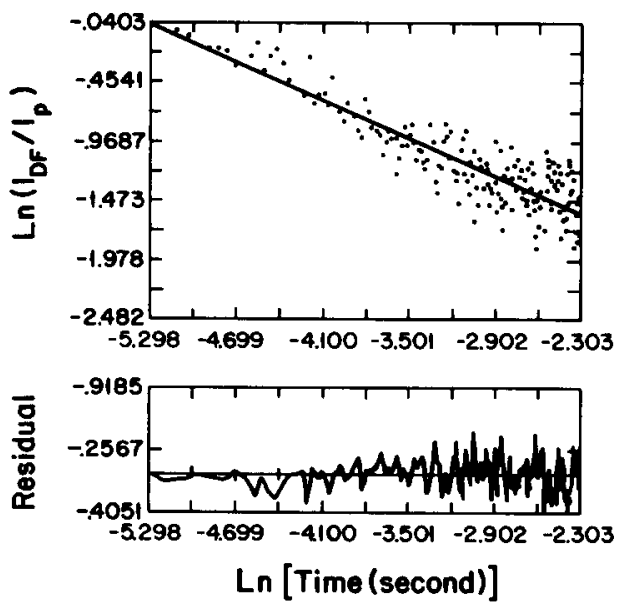

Fig. 1. $\operatorname{Ln}\left(I_{\mathrm{DF}} / I_{\mathrm{p}}\right)$ vs. $\ln [t(\mathrm{~s})]$ for a $0.01 \%$ sample.

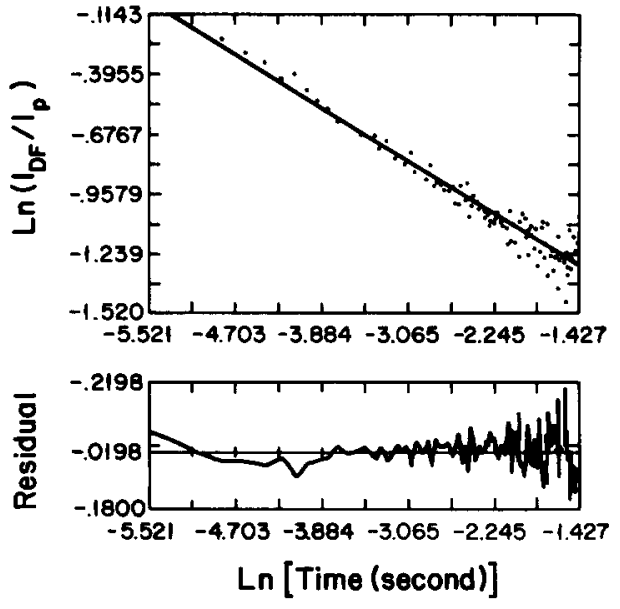

Fig. 2. $\operatorname{Ln}\left(I_{\mathrm{DF}} / I_{\mathrm{p}}\right)$ vs. $\ln [t(\mathrm{~s})]$ for a $50 \%$ sample.

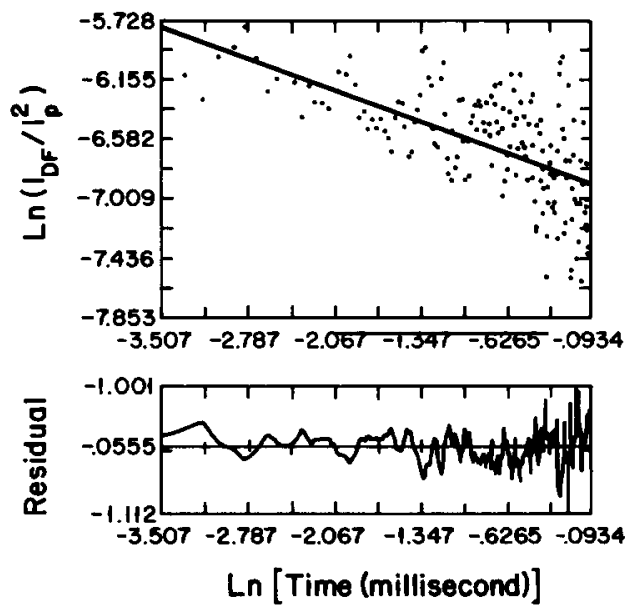

Fig. 3. $\operatorname{Ln}\left(I_{\mathrm{DF}} / I_{\mathrm{p}}^{2}\right)$ vs. $\ln [t(\mathrm{~ms})]$ for a $50 \%$ sample.

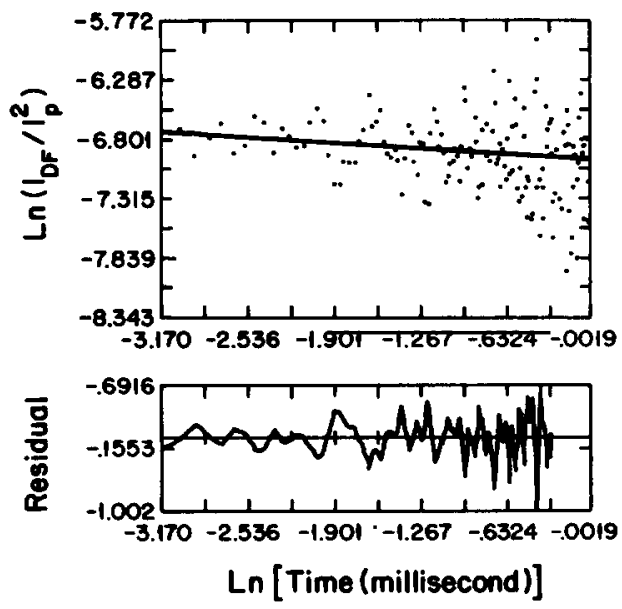

Fig. 4. $\operatorname{Ln}\left(I_{\mathrm{DP}} / I_{\mathrm{D}}^{2}\right)$ vs. $\ln [t(\mathrm{~ms})]$ for a $100 \%$ sample. 
tion transport, a random-coil chain has an effectively one-dimensional topology (as have most reasonable single-chain configurations). The major morphological conclusion for this low concentration sample is the absence of significant P1VN aggregation. For comparison we show a $50 \%$ sample (fig. 2) where $h=0.30 \pm 0.01$. Here we find a more complex transport topology. Clearly, even in this high concentration sample there is no total segregation, i.e., no break-up into domains of pure P1VN and domains of pure PMMA (note that all these samples were produced and maintained at or below room temperature). All this is corroborated by the short-time data (not enough time to populate excited defects or excimers). Here, instead of the pseudounimolecular kinetics (fusion of free and trapped excitations) leading to eq. (1), one finds bimolecular kinetics (homofusion of free excitations), leading to (1):

$I_{\mathrm{DF}}=k I_{\mathrm{p}}^{2}, \quad k=k_{0} t^{-h}$.

Figure 3 displays the short-time data for a $50 \%$ sample. The result is $h=0.30 \pm 0.04$, fully consistent with the fig. 2 results. Finally, fig. 4 displays the short-time data for the $100 \%$ P1VN sample. Now $h=0.01 \pm 0.01$. The complete mutual interpenetration of $\mathrm{P} 1 \mathrm{VN}$ chains in this sample implies an isotropic (homogeneous) excitation transport topology, for which $h=0$. From the morphological standpoint the difference between the
$50 \%$ and $100 \%$ samples is very interesting. If the $50 \%$ sample were totally segregated, domainwise, the results should be the same as for the $100 \%$ sample. We further note that between $0.01 \%$ and $40 \%$ the parameter $h$ varies non-monotonically. There is also a consistent behavior of $h$ with the singlet transport (monomer/ excimer ratio) data.

\section{Acknowledgements}

This research was supported in part by NSF Grant No. DMR 8801120 and in part by the Petroleum Research Fund administered by the American Chemical Society Grant No. 18791-AC5,6.

\section{References}

[1] R. Kopelman, Science 241 (1988) 1620.

[2] C.S. Li and R. Kopelman, Macromolecules, in press.

[3] C.S. Li and R. Kopelman, J. Phys. Chem., in press.

[4] E.I. Newhouse and R. Kopelman, Chem. Phys. Lett. 143 (1988) 106

[5] R. Kopelman, S.J. Parus, Z-Y. Shi, in: Dynamical Processes in Condensed Molecular Systems, eds. J. Klafter, J. Jortner and A. Blumen (World Scientific, Singapore, 1989) p. 231.

[6] J. Prasad and R. Kopelman, Phys. Rev. Lett. 58 (1987) 284. 